\title{
Black hole microstates from branes at angle
}

\section{Lorenzo Pieri}

Università di Roma Tor Vergata and I.N.F.N, Dipartimento di Fisica, Via della Ricerca Scientifica, I-00133 Rome, Italy

Centre for Research in String Theory, School of Physics and Astronomy,

Queen Mary University of London,

Mile End Road, London, E1 4NS, U.K.

E-mail: lorenzo.pieri@roma2.infn.it

ABSTRACT: We derive the leading $g_{s}$ perturbation of the SUGRA fields generated by a supersymmetric configuration of respectively 1, 2 or 4 D3-branes intersecting at an arbitrary angle via the computation of the string theory disk scattering amplitude of one massless NSNS field interacting with open strings stretched between the branes. The configuration with four branes is expected to be relevant for black hole microstate counting in four dimensions.

Keywords: Black Holes in String Theory, D-branes

ARXiv EPRINT: 1610.06156 


\section{Contents}

1 Introduction and conclusion $\quad 1$

2 One boundary amplitude $\quad 3$

3 Vertex operators for branes at angle 5

4 Two boundary amplitude $\quad 6$

5 Four boundary amplitude $\quad 8$

$\begin{array}{lll}5.1 & \text { Fermions in extended space-time dimensions } & 10\end{array}$

5.2 Fermions in compact dimensions 11

6 Comments on the supergravity solution 12

$\begin{array}{ll}\text { A Notations and conventions } & 14\end{array}$

B OPEs and correlators $\quad 16$

$\begin{array}{lll}\text { B.1 How to fix the normalization } & 17\end{array}$

$\begin{array}{ll}\text { C Rotation matrix for tilted branes } & 17\end{array}$

$\begin{array}{ll}\text { D Two boundary amplitude } & \mathbf{1 7}\end{array}$

$\begin{array}{ll}\text { D.1 Fermions in extended space-time dimensions } & 18\end{array}$

$\begin{array}{lll}\text { D.2 Fermions in compact dimensions } & 20\end{array}$

\section{Introduction and conclusion}

In a recent paper was explicitly uncovered an open-closed dictionary between microstates in string theory arising from open string stretched between D-branes and microstate geometries in supergravity for a system of 4 perpendicularly intersecting D3 branes [1]. This configuration of branes is particularly interesting, since it can be seen as the weak $g_{s}$ coupling picture of a four dimensional black holes with non vanishing area in the strong coupling (SUGRA) picture and computation of its degeneracy should led to the microscopic calculation of the entropy $[2,3]$.

We stress that for microstate geometries one means SUGRA solutions with the same asymptotic charges as the corresponding black hole, but differing from it near the location of the black hole horizon. The basic idea is that the singular black hole arises as a coarsegrained description of the underlying regular microstates when we trace out the region inside the would-be horizon, that is where the microstates differ from each other. The fact 
that these microstates can be found directly in SUGRA is the statement of the fuzzball proposal in a nutshell [4]. More precisely, the strongest form of the conjecture says that every string microstates is associated bijectively to regular and horizon-less microstates geometries. The beauty of this proposal lies in the possibility of solving the information loss paradox with tools already available, namely using mainly supergravity, branes and perturbative string theory. Of course a classical SUGRA solution must be thought as a very special state (coherent state) of the real fully quantum picture, therefore in order to completely describe the features of a quantum black hole, a better knowledge of non perturbative string theory will be required. Nevertheless, we could hope to catch the relevant physics in this approximation.

Much work has been done on 2 and 3 charge black holes, see for instance [5-12], but very little is known for the four charge case, corresponding to black holes in four dimensions (apparently the dimension in which we live). In this paper we generalize the work done in [1] by tilting the branes with some arbitrary angle, but still keeping the system supersymmetric. These configurations are relevant if one wants to study orbifold compactifications rather than the simple toroidal ones, leading to more realistic black hole models.

The strategy of the paper consists in deriving the leading $g_{s}$ perturbation of the SUGRA fields generated by the configuration of tilted branes via the computation of the string theory disk ${ }^{1}$ scattering amplitude of one massless NSNS field interacting with open strings binding the different branes. We begin the stringy computations by evaluating the amplitude for the emission of one closed NSNS field in the presence of one single stack of branes (section 1). To compute the amplitude with two different stacks (section 3) bound by open strings we first construct the open string vertex operators associated to every relevant pair of branes taken into account (section 2), paying attention to be consistent with the supersymmetry preserved by the system. Finally we perform the calculation for the 4 brane system (section 4), that it's the novel result of this letter.

Explicitly, once the amplitude $\mathcal{A}$ has been computed, to extract the leading perturbation is sufficient to use the effective field theory formula

$$
\delta \tilde{\Phi}(k)=\left(-\frac{\mathrm{i}}{k^{2}}\right) \frac{\delta \mathcal{A}(k)}{\delta \Phi}
$$

In [1] explicit agreement was shown between the stringy calculations and a known extremal SUGRA solution, in line with the idea that these classical solutions can account for the microstates of the corresponding black hole. This was achieved by expanding the SUGRA solutions at first order in $g_{s}$ and matching them with perturbations found from the formula (1.1). On the contrary, the emission found here are completely new and at the moment there is no known SUGRA solution in literature written in terms of arbitrary harmonic functions that can accommodate for the microstates found in string theory. We conjecture that such SUGRA solutions should exist and we give some arguments (section 5) supporting this thesis. It would be relevant to write explicitly such solution and we plan to address this issue in a future work.

\footnotetext{
${ }^{1}$ The disk is the worldsheet surface associated to the leading $g_{s}$ contribution of a mixed open-closed amplitudes.
} 
Two important points were left open in [1] and are not closed here. First of all, since the number of disk with different boundaries grows with the product of the charges like $Q_{1} Q_{2} Q_{3} Q_{4}$ and the SUGRA solution is dual to this configuration, we expect that there should be some way to compute the entropy directly from gravity, on the same line of previous successes for the D1D5 case $[13,14]$, without dealing with the complicated $\mathcal{N}=4$ quantum mechanics on the branes worldvolume. ${ }^{2}$ Second, to obtain the fuzzball of a black hole it is necessary to show that regular, horizonless and asymptotically flat solutions exist, and this means finding the explicit form of the harmonic function, similarly to what has been done in [16] for $A d S_{2} \times S_{2}$ asymptotics. We plan to also come back on this issues in a future work.

\section{One boundary amplitude}

In this section we compute the disk amplitude for the emission of one closed NSNS field from a single stack of Dp-branes at angles, ${ }^{3}$ in the weak coupling regime. By using (1.1) we will be able to extract the corresponding gravitational background generated in the SUGRA limit.

This calculation has already been performed in [1] for perpendicular intersecting branes. The procedure is identical for tilted branes, the only difference is the expression of the reflection matrix $R$; therefore in this section we simply state the result of the amplitude and setup notation for the next sections. The relevant vertex operators are

$$
\begin{aligned}
W_{N S N S}(z, \bar{z}) & =(E R)_{M N} e^{-\varphi} \psi^{M} e^{i k X}(z) e^{-\varphi} \psi^{N} e^{i k R X}(\bar{z}) \\
V_{\xi(\phi)}\left(x_{a}\right) & =\sum_{n=0}^{\infty} \xi_{i_{1} \ldots i_{n}} \partial X^{i_{1}}\left(x_{1}\right) \prod_{a=2}^{n} \int_{-\infty}^{\infty} \frac{d x_{a}}{2 \pi} \partial X^{i_{a}}\left(x_{a}\right)
\end{aligned}
$$

where $E=h+b$ is the polarization, that encompasses both the graviton and the B-field, $R$ is the reflection matrix encoding the boundary conditions of the open strings, $\varphi$ is a scalar field that bosonize the superghost, and $\psi$ and $X$ are the worldsheet fermions and bosons respectively. While the first vertex describe closed strings, the second vertex stands for an arbitrary number of untwisted (with endpoints on the same stack of branes) open string scalar fields that can be inserted on the boundary, with some polarization $\xi_{i_{1} \ldots i_{n}}$. Remarkably, the insertion of these fields translates into the full multipole expansion of a generic harmonic function appearing in the SUGRA fields.

The amplitude to evaluate is:

$$
\mathcal{A}_{N S-N S, \xi(\phi)}=\left\langle c(z) c(\bar{z}) c\left(z_{1}\right)\right\rangle\left\langle W_{N S-N S}(z, \bar{z}) V_{\xi(\phi)}\right\rangle
$$

Left and right string sectors are related by:

$$
X_{\text {right }}^{M}=R_{N}^{M} X_{\text {left }}^{N} \quad \psi_{\text {right }}^{M}=R_{N}^{M} \psi_{\text {left }}^{N} \quad \varphi_{\text {right }}=\varphi_{\text {left }}
$$

\footnotetext{
${ }^{2}$ See for instance [15] for BPS state counting in a pure D-brane configuration, even though the calculation is performed in the limit of small charges, that is the opposite of the SUGRA limit.

${ }^{3}$ Since there is only one stack, the angle is between the branes and a fixed reference frame. This angle is actually meaningless in an uncompactified geometry, where it can be reabsorbed by a change of coordinates, while is meaningful and not completely arbitrary in a compact space.
} 
The ten dimensional reflection matrix enforce the boundary conditions for branes at angle and therefore contains a non trivial internal part in $T^{6}$ :

$$
R_{N}^{M}=\left(\begin{array}{ccccc}
1 & 0 & 0 & 0 & 0 \\
0 & -\mathbb{1}_{3 \times 3} & 0 & 0 & 0 \\
0 & 0 & R\left(\theta_{1}\right) & 0 & 0 \\
0 & 0 & 0 & R\left(\theta_{2}\right) & 0 \\
0 & 0 & 0 & 0 & R\left(\theta_{3}\right)
\end{array}\right)
$$

where we have introduced the two dimensional matrices derived in the appendix:

$$
R\left(\theta_{i}\right)=\left(\begin{array}{cc}
\cos \left(2 \theta_{i}\right) & \operatorname{sen}\left(2 \theta_{i}\right) \\
\operatorname{sen}\left(2 \theta_{i}\right) & -\cos \left(2 \theta_{i}\right)
\end{array}\right)
$$

The final result is:

$$
\mathcal{A}_{N S N S, \xi(\phi)}=(E R) \xi(k)
$$

where $E R=E_{M N} R^{N}{ }_{P} \eta^{P M}$ and $\xi(k)=\sum_{n=0}^{\infty} \xi_{i_{1} \ldots i_{n}} k^{i_{1}} \ldots k^{i_{n}}$.

The emitted field is the graviton, therefore using (1.1) and Fourier transforming (setting here and in the following $\xi(k)$ to a constant for simplicity) with

$$
\int \frac{d^{3} k}{(2 \pi)^{3}} \frac{1}{k^{2}} e^{i k x}=\frac{1}{4 \pi} \frac{1}{r}
$$

one finds that the deviation from flat space is given by the schematic form:

$$
h_{M N}=\frac{(\eta R)_{M N}}{r}
$$

where all the inessential constants have been absorbed into $\xi(k)$. Notice that the result is proportional to $g_{s}$, indeed the factor $g_{s}^{2}$ coming from the massless closed string propagator in (1.1) partially cancels with the $g_{s}^{-1}$ associated to the disk (open strings don't carry additional $g_{s}$ factors).

This result can be directly compared to the SUGRA metric

$$
d s^{2}=L^{-\frac{1}{2}}\left(-d t^{2}+\sum_{I=1}^{3} d \tilde{y}_{I}^{2}\right)+L^{\frac{1}{2}}\left(\sum_{I=1}^{3} d y_{I}^{2}+\sum_{i=1}^{3} d x_{i}^{2}\right)
$$

of D3 brane with tilted internal axes. Indeed (focusing on the first torus) performing the rotation in the internal tori as

$$
\begin{aligned}
& \tilde{y}_{1}^{\prime}=\cos (\theta) \tilde{y}_{1}-\sin (\theta) y_{1} \\
& y_{1}^{\prime}=\sin (\theta) \tilde{y}_{1}+\cos (\theta) y_{1}
\end{aligned}
$$

and expanding at the leading order in $g_{s}$ with the ansatz $L=1+\frac{g_{s}}{r}$ one finds:

$$
h_{M N} d x^{M} d x^{N}=\frac{g_{s}}{2}\left(d t^{2}+\sum_{i=1}^{3} d x_{i}^{2}+\cos (2 \theta) d y_{1}^{2}+2 \sin (2 \theta) d y_{1} d \tilde{y}_{1}-\cos (2 \theta) d \tilde{y}_{1}^{2}\right)
$$

having omitted a similar contribution from the other two internal torii. This expression matches what appears in (2.8) after reabsorbing the overall constants. 


\section{Vertex operators for branes at angle}

Boundary conditions imposed by intersecting branes at angle force the open strings stretched between the branes to have non integer mode expansions, therefore leading to vertex operators involving angle-dependent bosonic and fermionic twist field. The T-dual picture is given by open strings ending on D-branes with magnetic fields switched on along the world-volume. In the following we will construct a supersymmetric system of 4 D3 branes at angles and then we will identify the vertex operators corresponding to each pair of branes.

Every Dp-brane imposes some restrictions on the spinors $\epsilon_{L, R}$ parametrizing the SUSY transformations generated by $\epsilon_{L} Q_{L}+\epsilon_{R} Q_{R}$, where $Q_{L, R}$ are the supercharges (with the same $10 \mathrm{~d}$ chirality) of $I I B$ superstring theory. Solutions to these constraints count the number of unbroken supercharges in the system and when angles are taken into account one finds that if the branes are related by SU(3) rotations some supersymmetry is preserved [18]. In particular, we require that at least $\mathcal{N}=1$ is preserved between every couples of branes, leading to a condition on the (relative) angles:

$$
\theta_{1}+\theta_{2}+\theta_{3}=0 \quad \bmod 2 \pi
$$

where $\theta_{I}$ is the angle between two branes in the $\left\{y_{I}, \tilde{y}_{I}\right\}$ torus. To preserve $\mathcal{N}=2,(3.1)$ must be true and an angle must be zero, and to have $\mathcal{N}=4$ all the angles need to be zero.

One can verify that the system of figure 1 satisfies (3.1) for all the six possible pairs of branes. The convention is that a positive angle is taken counterclockwise from the horizontal $y$ direction, so for instance a right arrow stands for $\theta=0$, a left arrow for $\theta=\pi$ and up arrow for $\theta=\pi / 2$.

To be concrete, we construct the vertex operators for the fermionic open string stretched between the (ordered) pair $D 3_{0} D 3_{3}$ (for a more general discussion on vertex operators at angle see [19]). In the canonical $-\frac{1}{2}$ superghost picture the vertex operator is: ${ }^{4}$

$$
V_{\mu}^{\{03\}}\left(z_{n}\right)=e^{-\frac{\varphi}{2}} \mu_{\{03\}}^{\beta} S_{\beta}\left(\sigma_{1 / 2-\theta}^{(1) \dagger} e^{i \theta \varphi_{1}}\right)\left(\sigma_{1 / 2-\theta}^{(2)} e^{-i \theta \varphi_{2}}\right)\left(e^{-i \varphi_{3} / 2}\right) e^{i k_{n} X}
$$

where $S_{\beta}$ is an $\mathrm{SO}(1,3)$ spin field (see the appendix for notation and conventions) and $\mu^{\beta}$ is the fermionic polarization with Chan Paton indices not shown explicitly. In particular every polarization $\mu^{\beta}$ is actually a Chan-Paton matrix, for instance $\left(\mu_{\{03\}}^{a b}\right)^{\beta}$ refers to the open string stretched between the brane $a$ in the stack 0 and the brane $b$ in the stack 3 (the order is important).

A bosonic twist $\sigma_{\xi}^{(I)}$ field must be inserted for every $I$-torus $(I=1,2,3)$ in which the branes are not parallel. The twist field is angle dependent and the angle $\xi$ is computed by rotating the first brane, the $D 3_{0}$, to the second brane, with a dagger if the rotation is clockwise and without dagger if it is counterclockwise. Of course $\sigma_{\theta}^{\dagger}=\sigma_{1-\theta}$. Similarly, one must add a fermionic twist $e^{i\left(\xi-\frac{1}{2}\right) \varphi_{I}}$ for every torus. As a check one can verify that the conformal dimension ${ }^{5}$ of the vertex operator is equal to 1 and that the vertex is consistent

\footnotetext{
${ }^{4}$ The subscript $\mu$ in $V_{\mu}$ is a symbol for the polarization, not an index!

${ }^{5}$ The conformal dimension of a bosonic twist field is given by $h\left(\sigma_{\theta}\right)=\frac{1}{2} \theta(1-\theta)$. Other useful formulas are $h\left(e^{q \varphi}\right)=-\frac{1}{2} q^{2}-q$ and $h\left(e^{i \lambda \varphi}\right)=\frac{1}{2} \lambda^{2}$.
} 

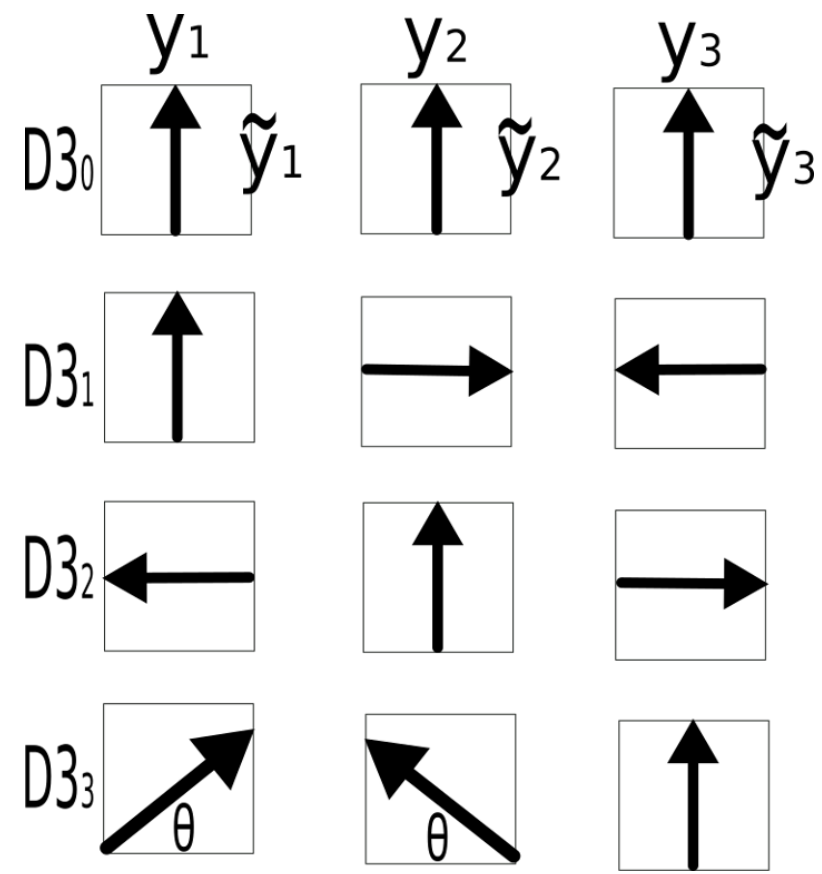

Figure 1. D3-brane configuration in the internal tori.

with the SUSY condition (3.1). Indeed the action of the $I I B$ positive chirality supercharge $Q^{(+++++)}=\oint d z e^{+\frac{i}{2}\left(\varphi_{4}+\varphi_{5}+\varphi_{1}+\varphi_{2}+\varphi_{3}\right)} e^{-\varphi / 2}$ must be well defined, implying that no noninteger powers of $z$ can appear in the commutator $\left[\epsilon Q, V_{\mu}^{(-1 / 2)}\right]$, which gives the susy-related bosonic vertex operator.

The opposite chirality fermion, the one stretched from $D 3_{3}$ to $D 3_{0}$ is recovered by doing the conjugation of the previous vertex:

$$
V_{\bar{\mu}}^{\{30\}}\left(z_{n}\right)=e^{-\frac{\varphi}{2}} \bar{\mu}_{\{30\}}^{\dot{\beta}} C_{\dot{\beta}}\left(\sigma_{1 / 2-\theta}^{(1)} e^{-i \theta \varphi_{1}}\right)\left(\sigma_{1 / 2-\theta}^{(2) \dagger} e^{i \theta \varphi_{2}}\right)\left(e^{i \varphi_{3} / 2}\right) e^{i k_{n} X}
$$

\section{Two boundary amplitude}

In this section we evaluate the disk amplitude for the scattering of one closed NSNS fields with two open strings stretched between two stacks of intersecting tilted D3 branes. This case already illustrates the crucial features that will be present in the more physically interesting four boundary computation. Similar calculation without non trivial angles have already been performed for $D 1 D 5$ and $D 3 D 3^{\prime}$ perpendicularly intersecting system in $[1,20,21]$. The system considered is a non threshold bound state of D-branes, meaning that the solution is not in a naive BPS superposition and individual branes cannot be freely separated (Higgs branch). From the microscopical point of view, this is signaled by the presence of a non zero open string condensate, or in other words of a non zero v.e.v. for the fields associated to open strings stretched between different branes.

The string amplitude to be computed is

$$
\mathcal{A}_{\mu \bar{\mu} N S N S}=\int \frac{d^{4} z}{V_{C K V}}\left\langle V_{\mu}^{(-1 / 2)}\left(z_{1}\right) V_{\bar{\mu}}^{(-1 / 2)}\left(z_{2}\right) W_{N S N S}^{(0,-1)}\left(z_{3}, z_{4}\right)\right\rangle
$$




\begin{tabular}{|c|c|c|c|c|c|c|c|c|c|c|}
\hline Brane & $\mathrm{t}$ & $x_{1}$ & $x_{2}$ & $x_{3}$ & $y_{1}$ & $\tilde{y}_{1}$ & $y_{2}$ & $\tilde{y}_{2}$ & $y_{3}$ & $\tilde{y}_{3}$ \\
\hline$D 3$ & - & $\cdot$ & $\cdot$ & $\cdot$ & $\cdot$ & - & $\cdot$ & - & $\cdot$ & - \\
$D 3^{\prime}$ & - & $\cdot$ & $\cdot$ & $\cdot$ & $\theta$ & & $\pi-\theta$ & & $\cdot$ & - \\
\hline
\end{tabular}

Table 1. D3-brane configuration: Neumann and Dirichlet directions are lines and dots respectively. The angle is taken with respect to the non tilded y coordinate.

in which $V_{C K V}$ is the conformal Killing volume, $z_{1}$ and $z_{2}$ are real, while $z_{3}=\bar{z}_{4}$ is complex.

The brane configuration can be taken to be the $D 3_{0} D 3_{3}$ as seen before. Here we rewrite the vertex operators needed for the calculation, with a superscript indicating the superghost charge:

$$
\begin{aligned}
V_{\mu}^{(-1 / 2)}\left(z_{1}\right) & =\mu_{\alpha} S^{\alpha} e^{-\varphi / 2}\left(\sigma_{1 / 2-\theta}^{(1) \dagger} e^{i \theta \varphi_{1}}\right)\left(\sigma_{1 / 2-\theta}^{(2)} e^{-i \theta \varphi_{2}}\right)\left(e^{-i \varphi_{3} / 2}\right) e^{i k_{1} X} \\
V_{\bar{\mu}}^{(-1 / 2)}\left(z_{2}\right) & =\bar{\mu}_{\dot{\alpha}} C^{\dot{\alpha}} e^{-\varphi / 2}\left(\sigma_{1 / 2-\theta}^{(1)} e^{-i \theta \varphi_{1}}\right)\left(\sigma_{1 / 2-\theta}^{(2) \dagger} e^{i \theta \varphi_{2}}\right)\left(e^{i \varphi_{3} / 2}\right) e^{i k_{2} X} \\
W_{N S N S}^{(0,-1)}\left(z_{3}, z_{4}\right) & =(E R)_{M N}\left(\partial X^{M}-i k \psi \psi^{M}\right) e^{i k X}\left(z_{3}\right) e^{-\varphi} \psi^{N} e^{i k R X}\left(z_{4}\right)
\end{aligned}
$$

The reflection matrix can be choosen to be the one associated to the $D 3_{0}$ or the one associated to the $D 3_{3}$, the final result will be independent of this choice. The spinorial structure of the condensate $v$ is:

$$
\mu^{\alpha} \bar{\mu}^{\dot{\beta}}=\left(\bar{\sigma}^{\mu}\right)^{\dot{\beta} \alpha} v_{\mu}
$$

The open string condensate microscopically encodes the different microstates of the black hole geometries. Notice that the condensate $v$ can be linked to the profile function $f(v)$ appearing as a displacement of the center of the $D 1 D 5$ fuzzball harmonic functions or alternatively as the profile of the oscillation of the string in the U-dual F1-P (fundamental string with momentum) system [22-26]. More precisely the condensate is linked to the derivative of $f(v)$ [20], therefore in some sense it gives the dynamic part of the displacement, whether the fixed part can be encoded in the untwisted scalars, as we have already said. While here the condensate is an arbitrary real number, in a proper treatment based on the microscopic theory on the world-volume of the D-branes it should be possible to quantize it, matching all the possible open string configuration to the respective condensate.

In the following we focus on constants open string fields, therefore we can consistently take all the open strings momenta to be vanishing. As we will see, care is needed in taking the zero momentum limit, indeed the amplitude is irreducible only if the disk diagram cannot factorize into two or more disk diagrams connected by propagating massless open fields. If the diagram factorize, the amplitude has massless open string poles at zero momentum exchanged; the associated worldsheet integral is divergent and there is no corresponding SUGRA emission. The closed string momentum satisfies $k^{2}=0$ and $k^{M} E_{M N}=0$, moreover we concentrate on a purely spatial momentum $k^{M}=\left\{k^{0}, k^{i}, k^{\text {int }}\right\}=\left\{0, k^{i}, 0\right\}$, analytically continuing the momentum to complex values in order to be consistent with the mass-shell condition.

Here we report directly the result of the computation, the reader interested in the details can found the explicit derivation in the appendix. The non compact part and the 
$(M, N)=(\overline{3}, 3)$ component have a finite amplitude given by:

$$
\begin{aligned}
& \mathcal{A}_{\mu \bar{\mu} N S N S}^{N C}=\frac{\pi}{\sqrt{2}}(E R)(k v) \\
& \mathcal{A}_{\mu \bar{\mu} N S N S}^{3 \overline{3}}=\frac{-\pi}{\sqrt{2}}(E R)_{[3 \overline{3}]}(k v)
\end{aligned}
$$

with $v k=v^{i} k^{i}$, and $(\overline{3}, 3)$ being the complex directions defined from the real ones $(\tilde{3}, 3)$. On the contrary the tilted directions give rise to divergent or vanishing amplitudes, depending on the symmetry property of the polarizations. The divergent part require some clarification: it implies that there is no associated SUGRA emission for that choice of the condensate. What's happening can be understood clearly from the $\mathrm{SO}(1,5)$ picture of the directions $\left\{t, x_{1}, x_{2}, x_{3}, y_{3}, \tilde{y}_{3}\right\}$ : the fermionic condensate $\mu^{A} \bar{\mu}^{B}$, where $A, B=1, \ldots, 4$, factorizes as $\mathbf{4} \times \mathbf{4}=\mathbf{6}+\mathbf{1 0}$. The $\mathbf{6}$, the anti-symmetric part of the product, is simply the vectorial representation, corresponding to the $\Gamma_{[A B]}^{\hat{M}}(\hat{M}=1, \ldots, 6)$ appearing in the fermionic kinetic term in the effective lagrangian, therefore giving a factorization channel for the $N S N S$-fermion-fermion diagram into two diagrams linked by a massless scalar field $\phi_{[A B]}$. To get a non factorizable diagram, one must restrict to the symmetric part of the product for which no fields with suitable indices are present.

A simple way to choose the symmetric part is to restrict to $S^{A}=S^{B}=e^{\frac{i}{2}\left(-\varphi_{3}-\varphi_{4}-\varphi_{5}\right)}$. The vertices to employ are then:

$$
\begin{aligned}
& V_{\mu}^{(-1 / 2)}\left(z_{1}\right)=\mu_{A} S^{A} e^{-\varphi / 2}\left(\sigma_{1 / 2-\theta}^{(1) \dagger} e^{i \theta \varphi_{1}}\right)\left(\sigma_{1 / 2-\theta}^{(2)} e^{-i \theta \varphi_{2}}\right) e^{i k_{1} X} \\
& V_{\bar{\mu}}^{(-1 / 2)}\left(z_{2}\right)=\bar{\mu}_{B} S^{B} e^{-\varphi / 2}\left(\sigma_{1 / 2-\theta}^{(1)} e^{-i \theta \varphi_{1}}\right)\left(\sigma_{1 / 2-\theta}^{(2) \dagger} e^{i \theta \varphi_{2}}\right) e^{i k_{2} X}
\end{aligned}
$$

resulting in the $\mathrm{SO}(1,5)$ amplitude:

$$
\mathcal{A}_{\mu \bar{\mu} N S N S}^{\mathrm{SO}(1,5)}=\frac{1}{3 !}(E R)_{\hat{M} \hat{N}} k_{\hat{P}} v^{\hat{M} \hat{N} \hat{P}}
$$

having defined a three index condensate contracting $\Gamma_{(A B)}^{\hat{M} \hat{N} \hat{P}}$ with $\mu^{(A} \bar{\mu}^{B)}$ and having used hatted $\mathrm{SO}(1,5)$ indices. Notice that in the final results the angles disappeared altogether. In order to see a non trivial angular dependence one must break the $\mathrm{SO}(1,5)$ symmetry by tilting even the third torus, therefore dealing with three point correlation functions of twist fields. Such correlators will be present in the next section, resulting in an angular dependence of the amplitudes.

\section{Four boundary amplitude}

Finally we consider the $1 / 8$ BPS configuration of the 4 stack of branes oriented like in figure 1. The string amplitudes correspond to a disk with four different boundaries and polarizations $\mu$ chosen in a cyclic order starting and ending on $D 3_{0}$. The associated condensate is complex, meaning that one can run the cycle in two different directions using $\mu$ or $\bar{\mu}$ respectively.

The amplitude to be computed is:

$$
\mathcal{A}_{\mu^{4} N S N S}=\int \frac{d^{4} z}{V_{C K V}}\left\langle V_{\mu}^{(-1 / 2)}\left(z_{1}\right) V_{\mu}^{(-1 / 2)}\left(z_{2}\right) V_{\mu}^{(-1 / 2)}\left(z_{3}\right) V_{\mu}^{(-1 / 2)}\left(z_{4}\right) W_{N S N S}^{(0,0)}\left(z_{5}, z_{6}\right)\right\rangle
$$




\begin{tabular}{|c|c|c|c|c|c|c|c|c|c|c|}
\hline Brane & $\mathrm{t}$ & $x_{1}$ & $x_{2}$ & $x_{3}$ & $y_{1}$ & $\tilde{y}_{1}$ & $y_{2}$ & $\tilde{y}_{2}$ & $y_{3}$ & $\tilde{y}_{3}$ \\
\hline$D 3_{0}$ & - & $\cdot$ & $\cdot$ & $\cdot$ & $\cdot$ & - & $\cdot$ & - & $\cdot$ & - \\
$D 3_{1}$ & - & $\cdot$ & $\cdot$ & $\cdot$ & $\cdot$ & - & $\rightarrow$ & $\cdot$ & $\leftarrow$ & $\cdot$ \\
$D 3_{2}$ & - & $\cdot$ & $\cdot$ & $\cdot$ & $\leftarrow$ & $\cdot$ & $\cdot$ & - & $\rightarrow$ & $\cdot$ \\
$D 3_{3}$ & - & $\cdot$ & $\cdot$ & $\cdot$ & $\theta$ & & $\pi-\theta$ & & $\cdot$ & - \\
\hline
\end{tabular}

Table 2. D3-brane configuration: Neumann and Dirichlet directions are lines and dots respectively. The arrow distinguishes between the $\theta=0$ (right arrow) and $\theta=\pi$ (left arrow) case. The angle is taken with respect to the non tilded y coordinate.

with vertex operators constructed as previously explained:

$$
\begin{aligned}
V_{\mu}^{\{01\}}\left(z_{1}\right) & =e^{-\frac{\varphi}{2}} \mu_{\{01\}}^{\alpha} S_{\alpha}\left(e^{-i \varphi_{1} / 2}\right)\left(\sigma_{1 / 2}^{(2) \dagger}\right)\left(\sigma_{1 / 2}^{(3)}\right) e^{i k_{1} X} \\
V_{\mu}^{\{12\}}\left(z_{2}\right) & =e^{-\frac{\varphi}{2}} \mu_{\{12\}}^{\beta} S_{\beta}\left(\sigma_{1 / 2}^{(1)}\right)\left(\sigma_{1 / 2}^{(2)}\right)\left(e^{-i \varphi_{3} / 2}\right) e^{i k_{2} X} \\
V_{\mu}^{\{23\}}\left(z_{3}\right) & =e^{-\frac{\varphi}{2}} \mu_{\{23\}}^{\dot{\alpha}} C_{\dot{\alpha}}\left(\sigma_{\theta}^{(1)} e^{i \varphi_{1}(\theta+1 / 2)}\right)\left(\sigma_{1 / 2-\theta}^{(2)} e^{-i \theta \varphi_{2}}\right)\left(\sigma_{1 / 2}^{(3)}\right) e^{i k_{3} X} \\
V_{\mu}^{\{30\}}\left(z_{4}\right) & =e^{-\frac{\varphi}{2}} \mu_{\{30\}}^{\dot{\beta}} C_{\dot{\beta}}\left(\sigma_{1 / 2-\theta}^{(1)} e^{-i \theta \varphi_{1}}\right)\left(\sigma_{1 / 2-\theta}^{(2) \dagger} e^{i \theta \varphi_{2}}\right)\left(e^{i \varphi_{3} / 2}\right) e^{i k_{4} X} \\
W^{(0,0)}\left(z_{5}, z_{6}\right) & =c_{\mathrm{NS}}(E R)_{M N}\left(\partial X^{M}-i k \psi \psi^{M}\right) e^{\mathrm{i} k X}\left(z_{5}\right)\left(\partial X^{N}-i k R \psi \psi^{N}\right) e^{\mathrm{i} k R X}\left(z_{6}\right)
\end{aligned}
$$

It's convenient to take the choice $\mu_{\{01\}}^{(\alpha} \mu_{\{12\}}^{\beta)} \bar{\mu}_{\{23\}}^{(\dot{\alpha}} \bar{\mu}_{\{30\}}^{\dot{\beta})}$ for the condensate. This configuration lives in the $\left(\mathbf{3}_{L}, \mathbf{3}_{R}\right)$, that can be seen as a symmetric traceless tensor $v^{i j}$, and leads to non factorizable diagrams.

In particular let's fix:

$$
S_{\alpha}=S_{\beta}=e^{\frac{i}{2}\left(\varphi_{4}+\varphi_{5}\right)} \quad C_{\dot{\alpha}}=C_{\dot{\beta}}=e^{-\frac{i}{2}\left(\varphi_{4}-\varphi_{5}\right)}
$$

therefore the only way to saturate the +2 superghost charge in the $\{5, \overline{5}\}$ torus is taking the term with four fermions in the closed string vertex. We split the calculation in non-compact and compact directions. We start by computing the correlators common to both cases:

$$
\begin{aligned}
\left\langle c\left(z_{1}\right) c\left(z_{2}\right) c\left(z_{4}\right)\right\rangle & =z_{12} z_{24} z_{41} \\
\left\langle\prod_{j=1}^{4} e^{-\varphi / 2}\left(z_{j}\right)\right\rangle & =\prod_{i<j} z_{i j}^{-1 / 4}
\end{aligned}
$$

The twist fields correlator in the third torus is a two point function, while in the other torus we have a three and a four point function. The latter correlators in principle receive a classical contribution proportional to the area of the polygon formed by the intersecting branes, but it can be neglected in the SUGRA limit ([19, 27]). The explicit expressions for the quantum part are:

$$
\begin{aligned}
\left\langle\sigma_{1 / 2}^{(3)}\left(z_{1}\right) \sigma_{1 / 2}^{(3)}\left(z_{3}\right)\right\rangle & =z_{13}^{-\frac{1}{4}} \\
\left\langle\sigma_{1 / 2}^{(1)}\left(z_{2}\right) \sigma_{\theta}^{(1)}\left(z_{3}\right) \sigma_{1 / 2-\theta}^{(1)}\left(z_{4}\right)\right\rangle & =\left(4 \pi \Gamma_{\left\{\frac{1}{2}, \theta, \frac{1}{2}-\theta\right\}}\right)^{\frac{1}{4}} z_{23}^{-\frac{\theta}{2}} z_{24}^{+\frac{\theta}{2}-\frac{1}{4}} z_{34}^{-\frac{\theta}{2}+\theta^{2}} \\
\left\langle\sigma_{1 / 2}^{(2)}\left(z_{1}\right) \sigma_{1 / 2}^{(2)}\left(z_{2}\right) \sigma_{1 / 2-\theta}^{(2)}\left(z_{3}\right) \sigma_{1 / 2+\theta}^{(2)}\left(z_{4}\right)\right\rangle & =z_{12}^{-\frac{1}{4}} z_{34}^{-\frac{1}{4}+\theta^{2}}\left(\frac{z_{13} z_{24}}{z_{14} z_{23}}\right)^{\frac{1}{4}} \mathcal{I}^{-\frac{1}{2}}[w]
\end{aligned}
$$


where we have used

$$
\begin{aligned}
& \left\langle\sigma_{\alpha}\left(z_{1}\right) \sigma_{\beta}\left(z_{2}\right) \sigma_{\gamma}\left(z_{3}\right)\right\rangle=\left(4 \pi \Gamma_{\{\alpha, \beta, \gamma\}}\right)^{\frac{1}{4}} z_{12}^{-\alpha \beta} z_{13}^{-\alpha \gamma} z_{23}^{-\beta \gamma} \\
& \Gamma_{\alpha, \beta, \gamma}=\frac{\Gamma(1-\alpha) \Gamma(1-\beta) \Gamma(1-\gamma)}{\Gamma(\alpha) \Gamma(\beta) \Gamma(\gamma)} \\
& \left\langle\sigma_{1-a}\left(z_{1}\right) \sigma_{a}\left(z_{2}\right) \sigma_{1-b}\left(z_{3}\right) \sigma_{b}\left(z_{4}\right)\right\rangle=z_{12}^{-a(1-a)} z_{34}^{-b(1-b)}\left(\frac{z_{13} z_{24}}{z_{14} z_{23}}\right)^{\frac{1}{2}(a+b)-a b} \mathcal{I}^{-\frac{1}{2}}(w) \\
& \mathcal{I}[w]=\frac{1}{2 \pi}\left(B_{1}(a, b) G_{2}(w) H_{1}(1-w)+B_{2}(a, b) G_{1}(w) H_{2}(1-w)\right) \\
& B_{1}(a, b)=\frac{\Gamma(a) \Gamma(1-b)}{\Gamma(1+a-b)} \quad B_{2}(a, b)=\frac{\Gamma(b) \Gamma(1-a)}{\Gamma(1+b-a)} \\
& G_{1}(w)={ }_{2} F_{1}[a, 1-b ; 1 ; w] \quad G_{2}(w)={ }_{2} F_{1}[1-a, b ; 1 ; w] \\
& H_{1}(w)={ }_{2} F_{1}[a, 1-b ; 1+a-b ; w] \quad H_{2}(w)={ }_{2} F_{1}[1-a, b ; 1-a+b ; w] \\
& w=\frac{z_{12} z_{34}}{z_{13} z_{24}}=1-x
\end{aligned}
$$

where the last equality is valid after the gauge fixing

$$
z_{1}=-\infty \quad z_{2}=0 \quad z_{3}=x \quad z_{4}=1 \quad z_{5}=z \quad z_{6}=\bar{z}
$$

\subsection{Fermions in extended space-time dimensions}

Now we specialize to the amplitude with the choice $(M, N)=(4, \overline{4})$ : all the worldsheet fermions $\psi$ are in the extended space-time directions.

$$
\begin{aligned}
\left\langle e^{-i \varphi_{1} / 2}\left(z_{1}\right) e^{i \varphi_{1}(\theta+1 / 2)}\left(z_{3}\right) e^{-i \theta \varphi_{1}}\left(z_{4}\right)\right\rangle & =z_{13}^{-\frac{\theta}{2}-\frac{1}{4}} z_{14}^{\frac{\theta}{2}} z_{34}^{-\theta^{2}-\frac{\theta}{2}} \\
\left\langle e^{-i \theta \varphi_{2}}\left(z_{3}\right) e^{i \theta \varphi_{2}}\left(z_{4}\right)\right\rangle & =z_{34}^{-\theta^{2}} \\
\left\langle e^{-i \varphi_{3} / 2}\left(z_{2}\right) e^{i \varphi_{3} / 2}\left(z_{4}\right)\right\rangle & =z_{24}^{-\frac{1}{4}}
\end{aligned}
$$

The correlator of the four spin fields and four fermions fields after bosonization is:

$$
\left\langle e_{\left(z_{1}\right)}^{\frac{\mathrm{i}}{2}\left(\varphi_{4}+\varphi_{5}\right)} e_{\left(z_{2}\right)}^{\frac{\mathrm{i}}{2}\left(\varphi_{4}+\varphi_{5}\right)} e_{\left(z_{3}\right)}^{-\frac{\mathrm{i}}{2}\left(\varphi_{4}-\varphi_{5}\right)} e_{\left(z_{4}\right)}^{-\frac{\mathrm{i}}{2}\left(\varphi_{4}-\varphi_{5}\right)} e_{\left(z_{5}\right)}^{\mathrm{i}\left(\varphi_{4}-\varphi_{5}\right)} e_{\left(z_{6}\right)}^{\mathrm{i}\left(-\varphi_{4}-\varphi_{5}\right)}\right\rangle=\frac{1}{4} z_{12}^{\frac{1}{2}} z_{16}^{-1} z_{26}^{-1} z_{34}^{\frac{1}{2}} z_{35}^{-1} z_{45}^{-1}
$$

Using the gauge fixing (5.18), the remaining worldsheet triple integral is: ${ }^{6}$

$$
I_{4}=\int_{0}^{1} d x(-x)^{-\frac{1}{2}-\frac{\theta}{2}}(x-1)^{-\theta} \mathcal{I}^{-\frac{1}{2}}[1-x] \int_{\mathbb{C}^{+}} \frac{d^{2} z}{\bar{z}(1-x)(x-z)}
$$

that can be reduced to a single integration by computing the integral in the upper half of the complex plane (for instance using $z=r e^{i \alpha}$ and performing the $\mathrm{r}$ integration first):

$$
\int_{\mathbb{C}^{+}} \frac{d^{2} z}{\bar{z}(1-x)(x-z)}=-\frac{\pi \log (x)}{(x-1)}
$$

\footnotetext{
${ }^{6}$ Notice that by putting $\theta=0$ in one recover the case of perpendicular branes, involving the correlator of $4 \mathbb{Z}_{2}$ twist fields $[1]$. In particular $\mathcal{I}^{-\frac{1}{2}}[1-x]_{\theta=0}=\left({ }_{2} F_{1}\left[\frac{1}{2}, \frac{1}{2} ; 1 ; x\right]_{2} F_{1}\left[\frac{1}{2}, \frac{1}{2} ; 1 ; 1-x\right]\right)^{-1 / 2}$.
} 
The remaining integral must be performed numerically and is a finite overall constant that can be reabsorbed into the open string condensate. In the particular case of $\theta=0$ the integral is purely imaginary, but for a generic angle the result has a real part too.

Recombining all the results obtained so far one obtains the amplitude:

$$
\mathcal{A}_{\mu^{4} N S N S}^{N C}=(E R)_{4 \overline{4}} k_{i} k_{j} v^{i j}(\theta)
$$

where all the constants, group theory factors and the Chan-Paton trace over the polarizations have been reabsorbed into the symmetric traceless condensate $v^{i j}(\theta)$. The real and imaginary components of the amplitude contribute respectively to the symmetric and antisymmetric parts of $(E R)_{M \bar{M}}$, therefore for a generic angle and generic complex condensate the system will emit both the graviton and the B-field. ${ }^{7}$ The associated harmonic is given by $\frac{3 x_{j} x_{i}-\delta_{i j} r^{2}}{r^{5}}$, having used

$$
\int \frac{d^{3} k}{(2 \pi)^{3}} \frac{-i k_{j} k_{i}}{|k|^{2}} e^{i k x}=\frac{i}{4 \pi} \frac{3 x_{j} x_{i}-\delta_{i j}|x|^{2}}{|x|^{5}} .
$$

\subsection{Fermions in compact dimensions}

Now we focus on the choice $(M, N)=(J, \bar{J})$ with $J=1,2,3$. We have already computed the correlators (5.8) and (5.9). The correlator of spin fields and fermions is the same for all $J$ :

$$
\left\langle S_{(\alpha}\left(z_{1}\right) S_{\beta)}\left(z_{2}\right) C_{(\dot{\alpha}}\left(z_{3}\right) C_{\dot{\beta})}\left(z_{4}\right) \psi^{\mu}\left(z_{5}\right) \psi^{\nu}\left(z_{6}\right)\right\rangle=\frac{\left(z_{12} z_{34}\right)^{1 / 2} z_{56}}{2 \prod_{i=1}^{4}\left(z_{i 5} z_{i 6}\right)^{1 / 2}} \sigma_{\alpha \dot{\alpha}}^{(\mu} \sigma_{\beta \dot{\beta}}^{\nu)}
$$

In the following we list the remaining correlators and the final gauge fixed integrals.

- $(M, N)=(1, \overline{1})$

$$
\begin{aligned}
& \left\langle e_{\left(z_{1}\right)}^{-i \varphi_{1} / 2} e_{\left(z_{3}\right)}^{i \varphi_{1}(\theta+1 / 2)} e_{\left(z_{4}\right)}^{-i \theta \varphi_{1}} \psi_{\left(z_{5}\right)}^{1} \psi_{\left(z_{6}\right)}^{\overline{1}}\right\rangle=\frac{1}{2} z_{13}^{-\frac{\theta}{2}-\frac{1}{4}} z_{14}^{\frac{\theta}{2}} z_{34}^{-\theta^{2}-\frac{\theta}{2}} z_{15}^{-\frac{1}{2}} z_{16}^{\frac{1}{2}} z_{35}^{\frac{\theta}{2}+\frac{1}{2}} z_{46}^{-\frac{\theta}{2}-\frac{1}{2}} z_{45}^{-\theta} z_{46}^{\theta} z_{56}^{-1} \\
& \left\langle e^{-i \theta \varphi_{2}}\left(z_{3}\right) e^{i \theta \varphi_{2}}\left(z_{4}\right)\right\rangle=z_{34}^{-\theta^{2}} \\
& \left\langle e^{-i \varphi_{3} / 2}\left(z_{2}\right) e^{i \varphi_{3} / 2}\left(z_{4}\right)\right\rangle=z_{24}^{-\frac{1}{4}} \\
& I_{1}=\int_{0}^{1} d x(-x)^{-\frac{1}{2}-\frac{\theta}{2}}(x-1)^{-\theta} \mathcal{I}^{-\frac{1}{2}}[1-x] \int_{\mathbb{C}^{+}} \frac{d^{2} z(x-z)^{\theta}(x-\bar{z})^{\theta-1}}{|z|(1-z)^{\theta+1 / 2}(1-\bar{z})^{-\theta+1 / 2}} \\
& \text { - }(M, N)=(2, \overline{2}) \\
& \left\langle e^{-i \varphi_{1} / 2}\left(z_{1}\right) e^{i \varphi_{1}(\theta+1 / 2)}\left(z_{3}\right) e^{-i \theta \varphi_{1}}\left(z_{4}\right)\right\rangle=z_{13}^{-\frac{\theta}{2}-\frac{1}{4}} z_{14}^{\frac{\theta}{2}} z_{34}^{-\theta^{2}-\frac{\theta}{2}} \\
& \left\langle e^{-i \theta \varphi_{2}}\left(z_{3}\right) e^{i \theta \varphi_{2}}\left(z_{4}\right) \psi^{2}\left(z_{5}\right) \psi^{\overline{2}}\left(z_{6}\right)\right\rangle=\frac{1}{2} z_{34}^{-\theta^{2}} z_{35}^{-\theta} z_{36}^{\theta} z_{45}^{\theta} z_{46}^{-\theta} z_{56}^{-1} \\
& \left\langle e^{-i \varphi_{3} / 2}\left(z_{2}\right) e^{i \varphi_{3} / 2}\left(z_{4}\right)\right\rangle=z_{24}^{-\frac{1}{4}} \\
& I_{2}=\int_{0}^{1} d x(-x)^{-\frac{1}{2}-\frac{\theta}{2}}(x-1)^{-\theta} \mathcal{I}^{-\frac{1}{2}}[1-x] \int_{\mathbb{C}^{+}} \frac{d^{2} z(x-z)^{-\theta-1 / 2}(x-\bar{z})^{\theta-1 / 2}}{|z|(1-z)^{-\theta+1 / 2}(1-\bar{z})^{\theta+1 / 2}}
\end{aligned}
$$

${ }^{7}$ It's nevertheless possible to select the complex open string condensate in such way that only the graviton is emitted. 
- $(M, N)=(3, \overline{3})$

$$
\begin{aligned}
&\left\langle e^{-i \varphi_{1} / 2}\left(z_{1}\right) e^{i \varphi_{1}(\theta+1 / 2)}\left(z_{3}\right) e^{-i \theta \varphi_{1}}\left(z_{4}\right)\right\rangle=z_{13}^{-\frac{\theta}{2}-\frac{1}{4}} z_{14}^{\frac{\theta}{2}} z_{34}^{-\theta^{2}-\frac{\theta}{2}} \\
&\left\langle e^{-i \theta \varphi_{2}}\left(z_{3}\right) e^{i \theta \varphi_{2}}\left(z_{4}\right)\right\rangle=z_{34}^{-\theta^{2}} \\
&\left\langle e^{-i \varphi_{3} / 2}\left(z_{2}\right) e^{i \varphi_{3} / 2}\left(z_{4}\right) \psi^{3}\left(z_{5}\right) \psi^{\overline{3}}\left(z_{6}\right)\right\rangle=\frac{1}{2} z_{24}^{-\frac{1}{4}} z_{25}^{-\frac{1}{2}} z_{26}^{\frac{1}{2}} z_{45}^{\frac{1}{2}} z_{46}^{-\frac{1}{2}} z_{56}^{-1} \\
& I_{3}=\int_{0}^{1} d x(-x)^{-\frac{1}{2}-\frac{\theta}{2}}(x-1)^{-\theta} \mathcal{I}^{-\frac{1}{2}}[1-x] \int_{\mathbb{C}^{+}} \frac{d^{2} z}{z(1-\bar{z})|x-z|}
\end{aligned}
$$

In all these cases for a generic angle and generic complex condensate both the graviton and the B-field are emitted.

\section{Comments on the supergravity solution}

It's very common in supergravity to find extremal solutions parametrized by arbitrary harmonic functions. The prototypical example is the Reissner-Nordstrom: in the limit in which the horizons coincide, the solution is extremal and becomes part of the larger Majumdar-Papapetrou family of solutions. To our knowledge, even though there is a conspicuous literature on SUGRA bound states of branes at angles (see for instance [2832]) there is no extremal BPS solution expressed in terms of arbitrary harmonics functions for branes intersecting at angles. The arbitrary harmonics are crucial in order to interpret the solutions as excitations of the 'naive' intersection of branes and to match with the stringy worldsheet computations.

To be concrete, let's remember what happen in the case of perpendicular $D 3$ branes. The 'naive' solution for marginally (zero binding energy) bound states of intersecting susy branes can be obtained by the harmonic superposition rule [33, 34]. For instance, limiting for simplicity to the metric, the system of 4 perpendicular $D 3$ brane pictured in figure 1 for $\theta=0$ is given by:

$$
\begin{aligned}
d s^{2}= & \left(H_{0} H_{1} H_{2} H_{3}\right)^{-\frac{1}{2}} d t^{2}+\left(H_{0} H_{1} H_{2} H_{3}\right)^{\frac{1}{2}} \sum_{i=1}^{3} d x_{i}^{2}+\left(\frac{H_{2} H_{3}}{H_{0} H_{1}}\right)^{\frac{1}{2}} d y_{1}^{2}+\left(\frac{H_{0} H_{1}}{H_{2} H_{3}}\right)^{\frac{1}{2}} d \tilde{y}_{1}^{2} \\
& +\left(\frac{H_{0} H_{2}}{H_{1} H_{3}}\right)^{\frac{1}{2}} d y_{2}^{2}+\left(\frac{H_{1} H_{3}}{H_{0} H_{2}}\right)^{\frac{1}{2}} d \tilde{y}_{2}^{2}+\left(\frac{H_{0} H_{3}}{H_{1} H_{2}}\right)^{\frac{1}{2}} d y_{3}^{2}+\left(\frac{H_{1} H_{2}}{H_{0} H_{3}}\right)^{\frac{1}{2}} d \tilde{y}_{3}^{2}
\end{aligned}
$$

where $H_{A}=1+\frac{Q_{A}}{r}$.

The associated metric parametrized in terms of arbitrary harmonic functions has been given has been written in $[1,16]$. In particular in [1] it was given in terms of 8 harmonic functions $H_{a}=\left\{V, L_{I}, K_{I}, M\right\}$ :

$$
d s^{2}=-e^{2 U}(d t+w)^{2}+e^{-2 U} \sum_{i=1}^{3} d x_{i}^{2}+\sum_{I=1}^{3}\left[\frac{d y_{I}^{2}}{V e^{2 U} Z_{I}}+V e^{2 U} Z_{I} \tilde{e}_{I}^{2}\right]
$$


with

$$
\begin{aligned}
Z_{I} & =L_{I}+\frac{\left|\epsilon_{I J K}\right|}{2} \frac{K_{J} K_{K}}{V} \\
\mu & =\frac{M}{2}+\frac{L_{I} K_{I}}{2 V}+\frac{\left|\epsilon_{I J K}\right|}{6} \frac{K_{I} K_{J} K_{K}}{V^{2}} \\
e^{-4 U} & =Z_{1} Z_{2} Z_{3} V-\mu^{2} V^{2} \\
b_{I} & =\frac{K_{I}}{V}-\frac{\mu}{Z_{I}} \\
\tilde{e}_{I} & =d \tilde{y}_{I}-b_{I} d y_{I} \\
*_{3}^{\text {flat }} d w & =V d \mu-\mu d V-V Z_{I} d\left(\frac{K_{I}}{V}\right)
\end{aligned}
$$

This solution, that besides the metric includes the $C_{4}$ Ramond-Ramond field, reduces to the naive one if one chooses $L_{I}=H_{I}, V=H_{0}$ and $K_{I}=M=0$, but in principle it can account for the hairs of the $4 \mathrm{~d}$ black holes for a proper choice of the harmonic functions, as suggested by the matching with stringy microstates obtained in [1]. This is precisely what has been done in the case of the D1-D5 fuzzball [22], in which the harmonic functions are such that: a) far away from the fuzzball the metric resembles a black hole with same asymptotic charges, b) the metric has a 'throat' region in which it approximates the 'naive' solution, c) the fuzzball deviates strongly from the black hole geometry at the location of the would-be horizon and d) the geometry is non singular and horizonless.

In the case of branes at angles the 'naive' solution can be written, for instance specializing to the case of two $D 3$ branes tilted in the first two internal torii (the $D 3_{0} D 3_{3}$ pair in figure 1), as:

$$
\begin{aligned}
d s^{2}= & e^{2 U_{\theta}}\left(-d t^{2}+\sum_{I=1}^{2} d y_{I}^{2}+\sum_{I=1}^{3} d \tilde{y}_{I}^{2}+\right. \\
& \left.+\sum_{A=1,2} \frac{Q_{A}}{r}\left(\left[\left(R_{A}\right)_{a}^{1} d y_{a}\right]^{2}+\left[\left(R_{A}\right)_{a}^{2} d y_{a}\right]^{2}\right)\right)+e^{-2 U_{\theta}}\left(d y_{3}^{2}+\sum_{i=1}^{3} d x_{i}^{2}\right) \\
F_{5}= & d t \wedge d r \wedge d \tilde{y}_{3} \wedge \partial_{r}\left[e ^ { 4 U _ { \theta } } \left(\sum_{A=1,2} \frac{Q_{A}}{r}\left[\left(R_{A}\right)_{a}^{\tilde{1}} d y_{a} \wedge\left(R_{A}\right)_{a}^{\tilde{2}} d y_{a}\right]-\right.\right. \\
& \left.\left.-\left(d y_{1} \wedge d y_{2}-d \tilde{y}_{1} \wedge d \tilde{y}_{2}\right) \frac{Q_{1} Q_{2}}{r^{2}} \sin ^{2}\left(\theta_{1}-\theta_{2}\right)\right)\right]+ \\
& +g e^{4 U_{\theta}} d \theta \wedge d \phi \wedge d y_{3} \wedge \partial_{r}\left[\sum_{A=1,2} \frac{Q_{A}}{r}\left[\left(R_{A}\right)_{a}^{1} d y_{a} \wedge\left(R_{A}\right)_{a}^{2} d y_{a}\right]\right]
\end{aligned}
$$

with $a=1, \tilde{1}, 2, \tilde{2}$ running only in the tilted torii (we define $d \tilde{y}_{1} \equiv d y_{\tilde{1}}$ ), $A=1,2$ labelling 
the two $D 3$ branes, $g$ the determinant of the metric and

$$
\begin{aligned}
e^{2 U_{\theta}}= & \left(1+\frac{Q_{1}+Q_{2}}{r}+\frac{Q_{1} Q_{2}}{r^{2}} \sin ^{2}\left(\theta_{1}-\theta_{2}\right)\right)^{-1 / 2} \\
R_{A}= & \left(\begin{array}{cccc}
\cos \left(\theta_{A}\right)-\sin \left(\theta_{A}\right) & 0 & 0 \\
\sin \left(\theta_{A}\right) & \cos \left(\theta_{A}\right) & 0 & 0 \\
0 & 0 & \cos \left(\theta_{A}\right) & \sin \left(\theta_{A}\right) \\
0 & 0 & -\sin \left(\theta_{A}\right) & \cos \left(\theta_{A}\right)
\end{array}\right)
\end{aligned}
$$

Even though the associated solution with arbitrary harmonics is still missing, we don't find any particular obstruction to it's existence. For instance after dimensional reduction on the internal $T^{6}$, focusing on the metric, the tilting of the internal torii translates in the presence of massless scalar fields grouped into the $h_{2,1}$ vector multiplets. Rotating the branes supersymmetrically is a continuous deformation, parametrized by some moduli fields, of a BPS system. In particular these moduli pertain to the deformations of the complex structure and although the attractor mechanism [35-37] fixes many of them in terms of the charges of the solution, in general some fields remains undetermined and they can account for the freedom to tilt the perpendicular brane configuration while still keeping the system supersymmetric.

A possibility is that the tilted solution can be obtained by a chain of boost and dualities from the perpendicular solution (6.2), in line with the results of [29].

\section{Acknowledgments}

A special thank to M. Bianchi and to J. F. Morales for suggestions and for reading the manuscript. We would also like to thank V. Balasubramanian, M. Costa, M. Cvetic, G. Dall'Agata, S. Giusto, R. Myers, N. Zinnato for suggestions and references.

\section{A Notations and conventions}

We will always take $\alpha^{\prime}=2$.

The index structure is the following:

- $M, N, R \ldots$ for 10-dimensional vector indices.

- $\hat{M}, \hat{N}, \hat{R} \ldots$ for 6 -dimensional vector indices.

- $\alpha, \beta \ldots$ for left spinorial indices in directions $\left\{t, x_{1}, x_{2}, x_{3}\right\}$

- $\dot{\alpha}, \dot{\beta} \ldots$ for right spinorial indices in directions $\left\{t, x_{1}, x_{2}, x_{3}\right\}$

- $A, B, C \ldots$ for $\mathrm{SO}(1,5)$ spinor indices. Upper position means right spinor, lower position left spinor.

- $\mu, \nu, \rho \ldots$ for vectorial indices in extended space-time directions $\left\{t, x_{1}, x_{2}, x_{3}\right\}$ 
- $i, j, k \ldots$ for vectorial indices in extended spatial directions $\left\{x_{1}, x_{2}, x_{3}\right\}$

- $1, \overline{1}, 2, \overline{2} \ldots$ for complex vectorial indices. In particular $\{1, \overline{1}\}$ parametrize the first internal torus $\left\{y_{1}, \tilde{y}_{1}\right\}$, whilst $\{4, \overline{4}\} \rightarrow\left\{t, x_{3}\right\},\{5, \overline{5}\} \rightarrow\left\{x_{1}, x_{2}\right\}$.

The left and right spin fields of directions $\left\{t, x_{1}, x_{2}, x_{3}\right\}$ are respectively bosonized by the scalar field $\varphi_{I}$ according to the formula:

$$
\begin{array}{rll}
\text { left }: & S_{\alpha}=e^{\frac{i}{2}\left( \pm \varphi_{4} \pm \varphi_{5}\right)} & \#(-)=\text { even } \\
\text { right }: & C_{\dot{\alpha}}=e^{\frac{i}{2}\left( \pm \varphi_{4} \pm \varphi_{5}\right)} & \#(-)=\text { odd }
\end{array}
$$

The complex fermions $\Psi$ are built from the real ones $\psi$ via $^{8}$

$$
\Psi^{I}=\frac{1}{\sqrt{2}}\left(\psi^{2 I-1}+i \psi^{2 I}\right) \quad \Psi^{\bar{I}}=\frac{1}{\sqrt{2}}\left(\psi^{2 I-1}-i \psi^{2 I}\right)
$$

and are bosonized as:

$$
\Psi^{I}=e^{i \varphi_{I}}
$$

In the text we will always use the notation $\psi$, even when referring to the complex fermion, as it should be clear from the context which of the two is used. Cocycle factors, needed to implement anticommutation, will be also omitted.

We follow [38] for the spinorial indices conventions. In particular we need the following formula:

$$
\begin{array}{rlrl}
\eta_{\mu \nu} & =(-,+,+,+) & & \epsilon^{21}=\epsilon_{12}=-1 \\
\epsilon^{12} & =\epsilon_{21}=+1 & & \sigma^{1}=\left(\begin{array}{ll}
0 & 1 \\
1 & 0
\end{array}\right) \\
\epsilon_{0123} & =-1 & & \\
\sigma^{0} & =\left(\begin{array}{cc}
-1 & 0 \\
0 & -1
\end{array}\right) & \sigma^{3}=\left(\begin{array}{cc}
1 & 0 \\
0 & -1
\end{array}\right) \\
\sigma^{2} & =\left(\begin{array}{cc}
0 & -i \\
i & 0
\end{array}\right) & \sigma^{2}=-\bar{\sigma}^{2}, \\
\sigma^{0} & =\bar{\sigma}^{0}, & & \\
\left(\sigma^{[\mu \nu]}\right)_{\alpha}{ }^{\beta} & \equiv \frac{1}{4}\left(\left(\sigma^{\mu}\right)_{\alpha \dot{\gamma}}\left(\bar{\sigma}^{\nu}\right)^{\dot{\gamma} \beta}-\left(\sigma^{\nu}\right)_{\alpha \dot{\gamma}}\left(\bar{\sigma}^{\mu}\right)^{\dot{\gamma} \beta}\right) & & \\
\left(\bar{\sigma}^{[\mu \nu]}\right)_{\dot{\beta}}^{\dot{\alpha}} & \equiv \frac{1}{4}\left(\left(\bar{\sigma}^{\mu}\right)^{\dot{\alpha} \gamma}\left(\sigma^{\nu}\right)_{\gamma \dot{\beta}}-\left(\bar{\sigma}^{\nu}\right)^{\dot{\alpha} \gamma}\left(\sigma^{\mu}\right)_{\gamma \dot{\beta}}\right) & &
\end{array}
$$

The four dimensional gamma matrices in the Weyl basis are:

$$
\Gamma^{\mu}=\left(\begin{array}{cc}
0 & \sigma^{\mu} \\
\bar{\sigma}^{\mu} & 0
\end{array}\right)
$$

\footnotetext{
${ }^{8} \mathrm{It}$ 's convenient to treat the torus $\{4, \overline{4}\} \rightarrow\left\{t, x_{3}\right\}$ in a different way by defining $\Psi^{4}=\frac{1}{\sqrt{2}}\left(\psi^{0}+\psi^{3}\right), \quad \Psi^{\overline{4}}=$ $\frac{1}{\sqrt{2}}\left(\psi^{0}-\psi^{3}\right)$ in order to use $\bar{\sigma}^{\mu}=\left\{\bar{\sigma}^{0}, \bar{\sigma}^{1}, \bar{\sigma}^{2}, \bar{\sigma}^{3}\right\}$. Otherwise one can use (A.3) and add an $i$ factor in front of $\bar{\sigma}^{3}$ in the definition of $\bar{\sigma}^{\mu}$.
} 
with associated charge conjugation matrix:

$$
\begin{aligned}
\left(\Gamma^{m}\right)^{T} & =-C\left(\Gamma^{m}\right) C^{-1} \\
C & =\left(\begin{array}{cc}
i\left(\sigma^{2} \bar{\sigma}^{0}\right)_{a}{ }^{b} & 0 \\
0 & i\left(\bar{\sigma}^{2} \sigma^{0}\right)^{\dot{c}} \\
\dot{d}
\end{array}\right)=\left(\begin{array}{cccc}
0 & -1 & 0 & 0 \\
1 & 0 & 0 & 0 \\
0 & 0 & 0 & 1 \\
0 & 0 & -1 & 0
\end{array}\right)=\left(\begin{array}{cc}
\epsilon_{a b} & 0 \\
0 & -\epsilon_{\dot{a} \dot{b}}
\end{array}\right)
\end{aligned}
$$

\section{B OPEs and correlators}

$$
\begin{aligned}
\left\langle\psi^{\mu}(z) \psi^{\nu}(w)\right\rangle & =\frac{\eta^{\mu \nu}}{(z-w)} \\
\left\langle X^{\mu}(z) X^{\nu}(w)\right\rangle & =-\eta^{\mu \nu} \log (z-w) \\
\left\langle\partial_{z} X^{\mu}(z) e^{i k X}(w)\right\rangle & =-\frac{i k^{\mu}}{(z-w)} \\
\left\langle\prod_{i=1}^{N} e^{q_{i} \varphi\left(z_{i}\right)}\right\rangle & =\prod_{i<j}^{N}\left(z_{i}-z_{j}\right)^{-q_{i} \cdot q_{j}} \quad \text { (superghost) } \\
\left\langle\prod_{i=1}^{N} e^{i \lambda_{i} \varphi\left(z_{i}\right)}\right\rangle & =\prod_{i<j}^{N}\left(z_{i}-z_{j}\right)^{+\lambda_{i} \cdot \lambda_{j}}
\end{aligned}
$$

We use the following basics OPEs in four dimensions:

$$
\begin{aligned}
C^{\dot{\alpha}}(z) S^{\beta}(w) & \sim-\frac{1}{\sqrt{2}}\left(\bar{\sigma}_{\mu}\right)^{\dot{\alpha} \beta} \psi^{\mu}(w) \\
\psi^{\mu} & \sim \frac{1}{\sqrt{2}}\left(\bar{\sigma}^{\mu}\right)^{\dot{\beta} \alpha} C_{\dot{\beta}} S_{\alpha} \\
S_{\alpha}(z) S_{\beta}(w) & \sim \frac{\epsilon_{\alpha \beta}}{(z-w)^{1 / 2}} \\
C_{\dot{\alpha}}(z) C_{\dot{\beta}}(w) & \sim-\frac{\epsilon_{\dot{\alpha} \dot{\beta}}}{(z-w)^{1 / 2}} \\
\psi^{m}(z) C^{\dot{\beta}}(w) & \sim \frac{1}{\sqrt{2}} \frac{\left(\bar{\sigma}^{m}\right)^{\dot{\beta} \alpha} S_{\alpha}(w)}{(z-w)^{1 / 2}} \\
\psi^{\mu}(z) S_{\alpha}(w) & \sim-\frac{1}{\sqrt{2}} \frac{\left(\sigma^{\mu}\right)_{\alpha \dot{\beta}} C^{\dot{\beta}}(w)}{(z-w)^{1 / 2}} \\
\psi^{\mu} \psi^{\nu}(z) S_{\alpha}(w) & \sim \frac{\left(\sigma^{\mu \nu}\right)_{\alpha}{ }^{\beta} S_{\beta}(w)}{(z-w)} \\
\psi^{\mu} \psi^{\nu}(z) C^{\dot{\alpha}}(w) & \sim \frac{\left(\bar{\sigma}^{\mu \nu}\right)_{\dot{\beta}}^{\dot{\alpha}} C^{\dot{\beta}}(w)}{(z-w)}
\end{aligned}
$$




\section{B.1 How to fix the normalization}

As an example, let's explictly fix the normalization of (B.6).

$$
\begin{aligned}
S^{1} & =e^{\frac{i}{2}\left(\varphi_{1}+\varphi_{2}\right)} & S^{2} & =e^{\frac{i}{2}\left(-\varphi_{1}-\varphi_{2}\right)} \\
C^{\dot{1}} & =e^{\frac{i}{2}\left(-\varphi_{1}+\varphi_{2}\right)} & C^{\dot{2}} & =e^{\frac{i}{2}\left(+\varphi_{1}-\varphi_{2}\right)}
\end{aligned}
$$

Therefore, choosing $\dot{\alpha}=\dot{1}, \beta=2$ and using (A.3):

$$
C^{\mathrm{i}}(z) S^{2}(w) \sim e^{-\varphi_{1}}=\Psi^{\overline{1}}=\frac{1}{\sqrt{2}}\left(\psi^{1}-i \psi^{2}\right)=N\left(\bar{\sigma}_{\mu}\right)^{\mathrm{i} 2} \psi^{\mu}
$$

So we have:

$$
N\left(\bar{\sigma}_{\mu}\right)^{\mathrm{i} 2} \psi^{\mu}=N\left(\psi^{1}\left(\bar{\sigma}_{1}\right)^{\mathrm{i} 2}+\psi^{2}\left(\bar{\sigma}_{2}\right)^{\mathrm{i} 2}\right)=N\left(\psi^{1}(-1)+\psi^{2}(i)\right)=-N\left(\psi^{1}-i \psi^{2}\right)
$$

From the comparison, it follows that $N=-\frac{1}{\sqrt{2}}$.

\section{Rotation matrix for tilted branes}

Consider two parallel branes in the $(x, y)$ plane. At the beginning the first and second brane are fixed in $y=0$, that is they are Neumann in $x$ and Dirichlet in $y$, where $X, Y$ are the open string coordinates in target space. This is equivalent to $\partial_{z} X=\partial_{\bar{z}} X$ and $\partial_{z} Y=-\partial_{\bar{z}} Y$ in complex coordinates. If we tilt a brane by an angle $\theta$ with respect to $y=0$, we need to impose $\partial_{z} X^{\prime}=\partial_{\bar{z}} X^{\prime}$ and $\partial_{z} Y^{\prime}=-\partial_{\bar{z}} Y^{\prime}$ where:

$$
\left(\begin{array}{l}
X^{\prime} \\
Y^{\prime}
\end{array}\right)=\left(\begin{array}{cc}
\cos (\theta) & -\sin (\theta) \\
\sin (\theta) & \cos (\theta)
\end{array}\right)\left(\begin{array}{l}
X \\
Y
\end{array}\right)
$$

After some algebra, for a counterclockwise rotation we find:

$$
\begin{aligned}
& \partial_{z} X^{\prime \mu}=R_{\nu}^{\mu} \partial_{\bar{z}} X^{\nu} \quad X^{1}=X, X^{2}=Y \\
& R(\theta)=\left(\begin{array}{cc}
\cos (2 \theta) & -\sin (2 \theta) \\
-\sin (2 \theta) & -\cos (2 \theta)
\end{array}\right)
\end{aligned}
$$

For a clockwise rotation we have:

$$
R(\theta)=\left(\begin{array}{cc}
\cos (2 \theta) & \sin (2 \theta) \\
\sin (2 \theta) & -\cos (2 \theta)
\end{array}\right)
$$

\section{Two boundary amplitude}

We split the calculation into two sections: the first one dealing with the amplitude with all the fermions in extended space-time directions and the second focusing on compact directions. Here we start by computing the correlators common to both sections:

$$
\begin{aligned}
\left\langle\sigma_{1 / 2-\theta}^{(1) \dagger}\left(z_{1}\right) \sigma_{1 / 2-\theta}^{(1)}\left(z_{2}\right)\right\rangle\left\langle\sigma_{1 / 2-\theta}^{(2)}\left(z_{1}\right) \sigma_{1 / 2-\theta}^{(2) \dagger}\left(z_{2}\right)\right\rangle & =z_{12}^{-\frac{1}{2}+2 \theta^{2}} \\
\left\langle e^{-\frac{1}{2} \varphi}\left(z_{1}\right) e^{-\frac{1}{2} \varphi}\left(z_{2}\right) e^{-\varphi}\left(z_{4}\right)\right\rangle & =z_{12}^{-\frac{1}{4}} z_{14}^{-\frac{1}{2}} z_{24}^{-\frac{1}{2}}
\end{aligned}
$$

The $\partial X$ piece in (4.4) gives vanishing contribution due to $k^{M} G_{M N}=0$. 


\section{D.1 Fermions in extended space-time dimensions}

The remaining internal part gives:

$$
\left\langle e^{i \theta \varphi_{1}} e^{-i \theta \varphi_{2}} e^{-i \varphi_{3} / 2}\left(z_{1}\right) e^{-i \theta \varphi_{1}} e^{i \theta \varphi_{2}} e^{i \varphi_{3} / 2}\left(z_{2}\right)\right\rangle=z_{12}^{-2 \theta^{2}-1 / 4}
$$

Taking $M=\mu$ and $N=\nu$, the last correlator, apart from overall constants, is:

$$
(k)_{\rho}(E R)_{\mu \nu}\left(\bar{\sigma}^{\nu}\right)^{\dot{\gamma} \delta}\left\langle S^{\alpha}\left(z_{1}\right) C^{\dot{\beta}}\left(z_{2}\right) J^{[\rho \mu]}\left(z_{3}\right) C_{\dot{\gamma}} S_{\delta}\left(z_{4}\right)\right\rangle
$$

in which we have defined the current $J^{[\rho \mu]}=: \psi^{\rho} \psi^{\mu}$ : and used the $\operatorname{SO}(1,3)$ relation $\psi^{\nu} \sim$ $\frac{1}{\sqrt{2}}\left(\bar{\sigma}^{\nu}\right)^{\dot{\gamma} \delta} C_{\dot{\gamma}} S_{\delta}$. Now we split the current in the dual (left) and anti-self dual part (right): ${ }^{9}$

$$
\begin{aligned}
J^{[\rho \mu]} & =J_{L}^{[\rho \mu]}+J_{R}^{[\rho \mu]} \\
J_{L}^{[\rho \mu]} & =\frac{1}{2}\left(J^{[\rho \mu]}+\frac{i}{2} \epsilon^{\rho \mu \tau \zeta} J_{[\tau \zeta]}\right) \\
J_{R}^{[\rho \mu]} & =\frac{1}{2}\left(J^{[\rho \mu]}-\frac{i}{2} \epsilon^{\rho \mu \tau \zeta} J_{[\tau \zeta]}\right)
\end{aligned}
$$

Using the fact that $\mathrm{SO}(1,3)$ Weyl spinors of different chirality have vanishing 2-point correlation function, we find:

$$
(k)_{\rho}(E R)_{\mu \nu}\left(\bar{\sigma}^{\nu}\right)^{\dot{\gamma} \delta}\left[\left\langle S_{\left(z_{1}\right)}^{\alpha}\left(J_{L}^{[\rho \mu]}\right)_{\left(z_{3}\right)}\left(S_{\delta}\right)_{\left(z_{4}\right)}\right\rangle\left(-\delta_{\dot{\gamma}}^{\dot{\beta}} z_{24}^{-1 / 2}\right)+\left\langle C_{\left(z_{2}\right)}^{\dot{\beta}}\left(\left(J_{R}^{[\rho \mu]}\right)_{\left(z_{3}\right)}\left(C_{\dot{\gamma}}\right)_{\left(z_{4}\right)}\right\rangle\left(\delta_{\delta}^{\alpha} z_{14}^{-1 / 2}\right)\right]\right.
$$

having used $\epsilon^{\alpha \beta} \epsilon_{\beta \delta}=\delta_{\delta}^{\alpha}$ and

$$
S_{\alpha}(z) S_{\beta}(w) \sim \frac{\epsilon_{\alpha \beta}}{(z-w)^{1 / 2}} \quad C_{\dot{\alpha}}(z) C_{\dot{\beta}}(w) \sim-\frac{\epsilon_{\dot{\alpha} \dot{\beta}}}{(z-w)^{1 / 2}}
$$

Conformal symmetry fixes the 3-point correlation functions of quasi-primary fields to be of the standard form

$$
\left\langle\Phi_{1}\left(z_{1}\right) \Phi_{2}\left(z_{2}\right) \Phi_{3}\left(z_{3}\right)\right\rangle=\frac{\mathcal{N}_{3 P t}}{z_{12}^{h_{1}+h_{2}-h_{3}} z_{23}^{h_{2}+h_{3}-h_{1}} z_{13}^{h_{1}+h_{3}-h_{2}}}
$$

Where $h$ is the conformal dimension of the fields and $\mathcal{N}_{3 P t}$ is a normalization $\mathcal{N}_{3 P t}$ constant. Using (A.11), (A.12) and that $h(J)=1, h(S)=h(C)=1 / 4$, we get: ${ }^{10}$

$$
\begin{aligned}
\left\langle S_{\left(z_{1}\right)}^{\alpha}\left(J_{L}^{[\rho \mu]}\right)_{\left(z_{3}\right)}\left(S_{\delta}\right)_{\left(z_{4}\right)}\right\rangle & =\frac{\left(\sigma^{[\rho \mu]}\right)_{\delta}{ }^{\alpha}}{z_{13} z_{14}^{-1 / 2} z_{34}} \\
\left\langle C_{\left(z_{2}\right)}^{\dot{\beta}}\left(J_{R}^{[\rho \mu]}\right)_{\left(z_{3}\right)}\left(C_{\dot{\gamma}}\right)_{\left(z_{4}\right)}\right\rangle & =-\frac{\left(\bar{\sigma}^{[\rho \mu]}\right)^{\dot{\beta}} \dot{\gamma}}{z_{23} z_{24}^{-1 / 2} z_{34}}
\end{aligned}
$$

\footnotetext{
${ }^{9}$ According to the convention $\star_{4} A^{\mu \nu} \equiv \frac{\mathrm{i}}{2} \epsilon^{\mu \nu \rho \sigma} A_{\rho \sigma}$.

${ }^{10}$ For instance the first correlator, in the group theory language of $s o(4) \sim s o(2) \oplus s o(2)$ is $\mathbf{2}_{L} \otimes(\mathbf{4} \otimes \mathbf{4})_{\text {asd }} \otimes$ $\mathbf{2}_{L}=\mathbf{2}_{L} \otimes\left(\mathbf{3}_{L}, \mathbf{1}_{R}\right) \otimes \mathbf{2}_{L}=\left(\mathbf{3}_{L}, \mathbf{1}_{R}\right) \otimes\left(\mathbf{1}_{L}+\mathbf{3}_{L}, \mathbf{1}_{R}\right)$ where $(\mathbf{4} \otimes \mathbf{4})_{\text {asd }}$ is the self dual antisymmetric part of the product and $\mathbf{4}=\left(\mathbf{2}_{L}, \mathbf{2}_{R}\right)$. Therefore the correlator has a singlet coming from $\left(\mathbf{3}_{L}, \mathbf{1}_{R}\right) \otimes\left(\mathbf{3}_{L}, \mathbf{1}_{R}\right)=$ $\mathbf{1}_{L}+\mathbf{3}_{L}+\mathbf{5}_{L}$. This means that the product is symmetric in the spinor indices (if properly lowered or raised) and antisymmetric in the vectorial indices.
} 
having used the self-duality/anti self-duality properties of the Lorentz generators. Now we employ the relations

$$
\begin{aligned}
\left(\bar{\sigma}^{\nu}\right)^{\dot{\beta} \delta}\left(\sigma^{[\rho \mu]}\right)_{\delta}{ }^{\alpha}=\frac{1}{2}\left[-i \epsilon^{\rho \mu \nu \eta}\left(\bar{\sigma}_{\eta}\right)^{\dot{\beta} \alpha}+\eta^{\mu \nu}\left(\bar{\sigma}^{\rho}\right)^{\dot{\beta} \alpha}-\eta^{\rho \nu}\left(\bar{\sigma}^{\mu}\right)^{\dot{\beta} \alpha}\right] \\
\left(\bar{\sigma}^{\nu}\right)^{\dot{\gamma} \alpha}\left(\bar{\sigma}^{[\rho \mu]}\right)_{\dot{\gamma}}^{\dot{\beta}}=\frac{1}{2}\left[-i \epsilon^{\rho \mu \nu \eta}\left(\bar{\sigma}_{\eta}\right)^{\dot{\beta} \alpha}-\eta^{\mu \nu}\left(\bar{\sigma}^{\rho}\right)^{\dot{\beta} \alpha}+\eta^{\rho \nu}\left(\bar{\sigma}^{\mu}\right)^{\dot{\beta} \alpha}\right]
\end{aligned}
$$

to obtain the integrand. Indeed putting everything together and gauge fixing to $z_{1}=-\infty$, $z_{2}=x, z_{3}=i, z_{4}=-i$ we get:

$$
\begin{aligned}
& \left\langle c\left(z_{1}\right) c\left(z_{3}\right) c\left(z_{4}\right)\right\rangle=z_{13} z_{14} z_{34} \\
& \mathcal{A}_{\mu \bar{\mu} N S N S}^{N C}=\mu_{\alpha} \bar{\mu}_{\dot{\beta}} \int_{-\infty}^{+\infty} d x\left(z_{13} z_{14} z_{34}\right) z_{12}^{-\frac{1}{2}+2 \theta^{2}} z_{12}^{-\frac{1}{4}} z_{14}^{-\frac{1}{2}} z_{24}^{-\frac{1}{2}} z_{12}^{-2 \theta^{2}-1 / 4} \\
& \times\left(\frac{i}{2 \sqrt{2}}\right)\left[\left(-i \epsilon^{\rho \mu \nu \eta}\left(\bar{\sigma}_{\eta}\right)^{\dot{\beta} \alpha}(k)_{\rho}(E R)_{\mu \nu}+(E R)(k \bar{\sigma})^{\dot{\beta} \alpha}-(\bar{\sigma} E R k)^{\dot{\beta} \alpha}\right) z_{13}^{-1} z_{34}^{-1} z_{14}^{1 / 2} z_{24}^{-1 / 2}+\right. \\
& \left.\left.\quad+\left(-i \epsilon^{\rho \mu \nu \eta}\left(\bar{\sigma}_{\eta}\right)^{\dot{\beta} \alpha}(k)_{\rho}(E R)_{\mu \nu}-(E R)(k \bar{\sigma})^{\dot{\beta} \alpha}+(\bar{\sigma} E R k)^{\dot{\beta} \alpha}\right) z_{23}^{-1} z_{24}^{1 / 2} z_{34}^{-1} z_{14}^{-1 / 2}\right)\right]
\end{aligned}
$$

Where $(E R)=(E R)_{\mu \nu} \eta^{\mu \nu}$ and $N C$ stands for non compact. The term $\bar{\sigma} E R k$ can be discarded if we are interested in a purely spatial (non internal) closed string momentum, using the fact that the momentum is transverse and the particular form of the reflection matrix in that directions, that is $k R=-k$. After some manipulations we obtain:

$$
\mathcal{A}_{\mu \bar{\mu} N S N S}^{N C}=\left(\frac{i}{2 \sqrt{2}}\right) \int_{-\infty}^{+\infty} d x\left[\left(-i \epsilon^{\rho \mu \nu \eta} v_{\eta}(k)_{\rho}(E R)_{\mu \nu}\right) \frac{2 x}{\left(1+x^{2}\right)}+(E R)(k v) \frac{-2 i}{\left(1+x^{2}\right)}\right]
$$

substituting the open string condensate $v^{\rho}=\mu_{\alpha} \bar{\mu}_{\dot{\beta}}\left(\bar{\sigma}^{\rho}\right)^{\dot{\beta} \alpha}$ and with $k v=k^{i} v^{i}$. The first part integrates to zero, while the second integral is simply: ${ }^{11}$

$$
\int_{-\infty}^{+\infty} \frac{d x}{\left(1+x^{2}\right)}=\pi
$$

Finally the amplitude of a massless closed string scattering on two tilted D3 branes is given by:

$$
\mathcal{A}_{\mu \bar{\mu} N S N S}^{N C}=\frac{\pi}{\sqrt{2}}(E R)(k v)
$$

\footnotetext{
${ }^{11}$ In all these calculations we have put the open strings momenta to zero from the beginning. If one wants to be rigorous, he should first do the full computation and then send all the open string momenta to zero. For instance the Koba-Nielsen factor, after using gauge fixing and kinematics of the momenta (and apart from an overall infinite constant due to $\left.z_{1}=-\infty\right)$ is:

$$
\left\langle e^{i k_{1} X}\left(z_{1}\right) e^{i k_{2} X}\left(z_{2}\right) e^{i k X}\left(z_{3}\right) e^{i k R X}\left(z_{4}\right)\right\rangle=\left|1+x^{2}\right|^{\alpha^{\prime} k_{2} k}|2 i|^{\alpha^{\prime} k R k / 2}
$$

Therefore the correct integral (D.18), using the integral beta function representation is [39]:

$$
\lim _{k 2 \rightarrow 0} \int_{-\infty}^{+\infty} \frac{d x}{\left(1+x^{2}\right)}\left|1+x^{2}\right|^{\alpha^{\prime} k_{2} k}=\lim _{k 2 \rightarrow 0} B\left(-\alpha^{\prime} k_{2} k+\frac{1}{2}, \frac{1}{2}\right)=\pi .
$$


Employing (1.1), one finds that the only emitted fields are the diagonal components of the metric and using

$$
\int \frac{d^{3} k}{(2 \pi)^{3}} \frac{-i k_{j}}{|k|^{2}} e^{i k x}=\frac{1}{4 \pi} \frac{x_{j}}{|x|^{3}}
$$

one finds that the scaling of the harmonic function is given by $\frac{x_{j}}{r^{3}}$.

\section{D.2 Fermions in compact dimensions}

One of the fermion of the closed string vertex still needs to be in the first four directions, in order to saturate the spin fields charge. The remaining fermions must be of opposite chirality: $(M, N)=(I, \bar{I})$ in $(4.1)$. We start with $(M, N)=(3, \overline{3})$ and the conjugate choice $(M, N)=(\overline{3}, 3)$. The correlators (D.1), (D.2) and (D.13) are unchanged. What is left is: ${ }^{12}$

$$
\begin{aligned}
(k)_{\rho}\left\langle S_{\left(z_{1}\right)}^{\alpha} C_{\left(z_{2}\right)}^{\dot{\beta}} \psi_{\left(z_{3}\right)}^{\rho}\right\rangle & =(k)_{\rho} \frac{1}{\sqrt{2}} \frac{\left(\bar{\sigma}^{\rho}\right)^{\dot{\beta} \alpha}}{z_{13}^{1 / 2} z_{23}^{1 / 2}} \\
\left\langle e^{i \theta \varphi_{1}} e^{-i \theta \varphi_{2}}\left(z_{1}\right) e^{-i \theta \varphi_{1}} e^{i \theta \varphi_{2}}\left(z_{2}\right)\right\rangle & =z_{12}^{-2 \theta^{2}} \\
\left\langle e^{-i \varphi_{3} / 2}\left(z_{1}\right) e^{i \varphi_{3} / 2}\left(z_{2}\right) e^{ \pm i \varphi_{3}}\left(z_{3}\right) e^{\mp i \varphi_{3}}\left(z_{4}\right)\right\rangle & =\frac{1}{2} z_{12}^{-\frac{1}{4}} z_{13}^{\mp \frac{1}{2}} z_{14}^{ \pm \frac{1}{2}} z_{23}^{ \pm \frac{1}{2}} z_{24}^{\mp \frac{1}{2}} z_{34}^{-1}
\end{aligned}
$$

Summing these two contributions and gauge fixing to $z_{1}=-\infty, z_{2}=x, z_{3}=i, z_{4}=-i$ we get:

$$
\mathcal{A}_{\mu \bar{\mu} N S N S}^{3 \overline{3}}=\left(\frac{i}{2 \sqrt{2}}\right) \int_{-\infty}^{+\infty} d x(k v)\left(\frac{(E R)_{3 \overline{3}}}{x-i}+\frac{(E R)_{\overline{3} 3}}{x+i}\right)
$$

that select the antisymmetric part of $(E R)_{3 \overline{3}}$, having used that $\int_{-\infty}^{+\infty} \frac{d x}{x-i}=\int_{-\infty}^{+\infty} \frac{d x(x+i)}{x^{2}+1}=$ $i \pi$. Since the reflection matrix is diagonal with $(-1,1)$ eigenvalues in the third torus, the emitted field is the metric.

$$
\mathcal{A}_{\mu \bar{\mu} N S N S}^{3 \overline{3}}=\frac{-\pi}{\sqrt{2}}(E R)_{[3 \overline{3}]}(k v)
$$

The emitted field is therefore the off-diagonal (real) metric in the third torus and the scaling of the harmonic function is the one found previously for $N C$ components.

If instead we compute the $(M, N)=(1, \overline{1})$ we find a divergent or vanishing integral for every angle. Indeed one finds:

$$
\mathcal{A}_{\mu \bar{\mu} N S N S}^{1 \overline{1}} \propto \int_{-\infty}^{+\infty} d x(x-i)^{-\theta-\frac{1}{2}}(x+i)^{\theta-\frac{1}{2}}
$$

that can be shown to be divergent using [40]:

$$
\int_{-\infty}^{+\infty} d x(x-i)^{a}(x+i)^{b}=-\pi(2 i)^{2+a+b} e^{-\pi i a} \frac{\Gamma(-1-a-b)}{\Gamma(-a) \Gamma(-b)}
$$

The same reasoning applies to $(M, N)=(2, \overline{2})$.

The calculation leading to (4.10) has been done in [1].

\footnotetext{
${ }^{12}$ Notice that we have simplified the calculation by employing only scalar fields in the non compact space, otherwise we should have computed excited twist fields coming from the correlators of scalars and twist fields.
} 
Open Access. This article is distributed under the terms of the Creative Commons Attribution License (CC-BY 4.0), which permits any use, distribution and reproduction in any medium, provided the original author(s) and source are credited.

\section{References}

[1] M. Bianchi, J.F. Morales and L. Pieri, Stringy origin of 4d black hole microstates, JHEP 06 (2016) 003 [arXiv: 1603.05169] [INSPIRE].

[2] A. Strominger and C. Vafa, Microscopic origin of the Bekenstein-Hawking entropy, Phys. Lett. B 379 (1996) 99 [hep-th/9601029] [INSPIRE].

[3] V. Balasubramanian and F. Larsen, On D-branes and black holes in four-dimensions, Nucl. Phys. B 478 (1996) 199 [hep-th/9604189] [INSPIRE].

[4] S.D. Mathur, The fuzzball proposal for black holes: an elementary review, Fortsch. Phys. 53 (2005) 793 [hep-th/0502050] [INSPIRE].

[5] S.D. Mathur, Fuzzballs and the information paradox: a summary and conjectures, arXiv:0810.4525 [INSPIRE].

[6] I. Bena and N.P. Warner, Black holes, black rings and their microstates, Lect. Notes Phys. 755 (2008) 1 [hep-th/0701216] [INSPIRE].

[7] K. Skenderis and M. Taylor, The fuzzball proposal for black holes, Phys. Rept. 467 (2008) 117 [arXiv: 0804.0552] [INSPIRE].

[8] S. Giusto, O. Lunin, S.D. Mathur and D. Turton, D1-D5-P microstates at the cap, JHEP 02 (2013) 050 [arXiv: 1211.0306] [INSPIRE].

[9] I. Bena, J. de Boer, M. Shigemori and N.P. Warner, Double, double supertube bubble, JHEP 10 (2011) 116 [arXiv:1107.2650] [inSPIRE].

[10] S. Giusto and R. Russo, Perturbative superstrata, Nucl. Phys. B 869 (2013) 164 [arXiv: 1211.1957] [INSPIRE].

[11] S. Giusto and R. Russo, Superdescendants of the D1-D5 CFT and their dual 3-charge geometries, JHEP 03 (2014) 007 [arXiv:1311.5536] [INSPIRE].

[12] I. Bena et al., Smooth horizonless geometries deep inside the black-hole regime, Phys. Rev. Lett. 117 (2016) 201601 [arXiv: 1607.03908] [INSPIRE].

[13] V.S. Rychkov, D1-D5 black hole microstate counting from supergravity, JHEP 01 (2006) 063 [hep-th/0512053] [INSPIRE].

[14] C. Krishnan and A. Raju, A note on D1-D5 entropy and geometric quantization, JHEP 06 (2015) 054 [arXiv: 1504.04330] [INSPIRE].

[15] A. Chowdhury, R.S. Garavuso, S. Mondal and A. Sen, Do all BPS black hole microstates carry zero angular momentum?, JHEP 04 (2016) 082 [arXiv: 1511.06978] [INSPIRE].

[16] O. Lunin, Bubbling geometries for $A d S_{2} \times S^{2}$, JHEP 10 (2015) 167 [arXiv:1507.06670] [INSPIRE].

[17] M. Bertolini, M. Billò, A. Lerda, J.F. Morales and R. Russo, Brane world effective actions for D-branes with fluxes, Nucl. Phys. B 743 (2006) 1 [hep-th/0512067] [INSPIRE].

[18] M. Berkooz, M.R. Douglas and R.G. Leigh, Branes intersecting at angles, Nucl. Phys. B 480 (1996) 265 [hep-th/9606139] [INSPIRE].

[19] M. Cvetič and R. Richter, Proton decay via dimension-six operators in intersecting D6-brane models, Nucl. Phys. B 762 (2007) 112 [hep-th/0606001] [INSPIRE]. 
[20] S. Giusto, J.F. Morales and R. Russo, D1-D5 microstate geometries from string amplitudes, JHEP 03 (2010) 130 [arXiv:0912.2270] [INSPIRE].

[21] S. Giusto, R. Russo and D. Turton, New D1-D5-P geometries from string amplitudes, JHEP 11 (2011) 062 [arXiv: 1108.6331] [INSPIRE].

[22] O. Lunin and S.D. Mathur, Metric of the multiply wound rotating string, Nucl. Phys. B 610 (2001) 49 [hep-th/0105136] [INSPIRE].

[23] O. Lunin and S.D. Mathur, AdS/CFT duality and the black hole information paradox, Nucl. Phys. B 623 (2002) 342 [hep-th/0109154] [INSPIRE].

[24] O. Lunin, S.D. Mathur and A. Saxena, What is the gravity dual of a chiral primary?, Nucl. Phys. B 655 (2003) 185 [hep-th/0211292] [INSPIRE].

[25] O. Lunin, J.M. Maldacena and L. Maoz, Gravity solutions for the D1-D5 system with angular momentum, hep-th/0212210 [INSPIRE].

[26] I. Kanitscheider, K. Skenderis and M. Taylor, Fuzzballs with internal excitations, JHEP 06 (2007) 056 [arXiv:0704.0690] [INSPIRE].

[27] M. Cvetič, R. Richter and T. Weigand, Computation of D-brane instanton induced superpotential couplings: Majorana masses from string theory, Phys. Rev. D 76 (2007) 086002 [hep-th/0703028] [INSPIRE].

[28] J.C. Breckenridge, G. Michaud and R.C. Myers, New angles on D-branes, Phys. Rev. D 56 (1997) 5172 [hep-th/9703041] [INSPIRE].

[29] K. Behrndt and M. Cvetič, BPS saturated bound states of tilted p branes in type-II string theory, Phys. Rev. D 56 (1997) 1188 [hep-th/9702205] [InSPIRE].

[30] M.S. Costa and M. Cvetič, Nonthreshold D-brane bound states and black holes with nonzero entropy, Phys. Rev. D 56 (1997) 4834 [hep-th/9703204] [INSPIRE].

[31] V. Balasubramanian, F. Larsen and R.G. Leigh, Branes at angles and black holes, Phys. Rev. D 57 (1998) 3509 [hep-th/9704143] [INSPIRE].

[32] M. Bertolini and M. Trigiante, Regular BPS black holes: macroscopic and microscopic description of the generating solution, Nucl. Phys. B 582 (2000) 393 [hep-th/0002191] [INSPIRE].

[33] A.A. Tseytlin, Harmonic superpositions of M-branes, Nucl. Phys. B 475 (1996) 149 [hep-th/9604035] [INSPIRE].

[34] J.P. Gauntlett, D.A. Kastor and J.H. Traschen, Overlapping branes in M-theory, Nucl. Phys. B 478 (1996) 544 [hep-th/9604179] [INSPIRE].

[35] S. Ferrara, R. Kallosh and A. Strominger, $N=2$ extremal black holes, Phys. Rev. D 52 (1995) R5412 [hep-th/9508072] [INSPIRE].

[36] S. Ferrara and R. Kallosh, Supersymmetry and attractors, Phys. Rev. D 54 (1996) 1514 [hep-th/9602136] [INSPIRE].

[37] S. Ferrara, G.W. Gibbons and R. Kallosh, Black holes and critical points in moduli space, Nucl. Phys. B 500 (1997) 75 [hep-th/9702103] [INSPIRE].

[38] J. Wess and J.A. Bagger, Supersymmetry and supergravity, $2^{\text {nd }}$ ed., Princeton Series in Physics, Princeton Univ. Press, Princeton NJ U.S.A., (1992) [InSPIRE].

[39] S. Stieberger, Open \& closed vs. pure open string disk amplitudes, arXiv:0907.2211 [INSPIRE].

[40] S. Stieberger and T.R. Taylor, Disk scattering of open and closed strings (I), Nucl. Phys. B 903 (2016) 104 [arXiv: 1510.01774] [INSPIRE]. 